MARYJO BRANQUINHO'

FERNANDA GERALDES ${ }^{2}$

FERNANDA ÁGUAS ${ }^{3}$

\title{
Impacto da terapia hormonal sobre o peso corpóreo
}

\section{Impact of hormone replacement therapy on body weight}

\section{Artigo original}

Palavras-chave

Menopausa Terapia de reposição hormonal Ganho de peso Índice de massa corporal

Peso corporal

Keywords

Menopause Hormone replacement therapy Weight gain

Body mass index Body weight

\section{Resumo}

OBJETIVO: avaliar o efeito da terapêutica hormonal (TH) no peso de mulheres na peri-menopausa, assim como o efeito de diferentes regimes terapêuticos no referido parâmetro. MÉTODOS: estudo retrospectivo de 139 mulheres, com menopausa há menos de 2 anos, acompanhadas na consulta de climatério do nosso departamento. Obtiveram-se dois grupos: mulheres a quem se iniciou TH $(n=89)$ e outro, grupo controle, sem terapia hormonal $(n=50)$. Em cada grupo, foi avaliada a modificação ponderal no intervalo de 1 ano após a primeira consulta. Nas submetidas a TH, avaliou-se esse mesmo parâmetro em função de diferentes regimes terapêuticos preconizados: estrogênio isolado vs estroprogestagênio e dose standard vs baixa dosagem. A análise estatística foi realizada com recurso ao programa SPSS ${ }^{\circledR}$, adotando-se como nível de significância valores $p<0,05$. RESULTADOS: os dois grupos foram semelhantes no que diz respeito a características basais e demográficas. No nosso estudo, constatou-se um aumento ponderal médio superior no grupo controle relativamente ao grupo de mulheres sob TH (434 vs 76 g), embora a diferença verificada não seja estatisticamente significativa $(p=0,406)$; Nas usuárias de $\mathrm{TH}$, aquelas sob estrogênio isolado tiveram um aumento ponderal acrescido face a mulheres sob terapêutica com estroprogestativo (775 vs $24 \mathrm{~g}$ ), embora com diferenças não significativas, o mesmo sucedendo quando analisada a dosagem de TH inicialmente prescrita 192 vs 49 g). CONCLUSÕES: apesar da crença comum do aumento ponderal associado à TH, os resultados do estudo descrito parecem contrariar esse aspecto, não havendo um ganho ponderal adicional ao normalmente associado a este período da vida da mulher.

\section{Abstract}

PURPOSE: to evaluate the effect of hormone replacement therapy $(H T)$ on the weight on perimenopausal women as well as the effect of different treatment regimens on this parameter. METHODS: a retrospective study of 139 women with menopause for less than 2 years, who were monitored with periodical visits in our department. We compared two groups: women who started HT (n=89) with women who had no hormonal treatment $(n=50)$ and in the two groups, we evaluated the changes in body weight over a 1 -year period. In the first group, we assessed the same parameter as a function of different treatment regimens: estrogen alone versus estrogen combined with progestin and standard dose versus low dose. The SPSS ${ }^{\circledR}$ program was used for statistical analysis, with the level of significance set at $p<0.05$. RESULTS: the groups were similar with respect to demographic and baseline characteristics; weight gain was higher in the untreated group (434 vs $76 \mathrm{~g})$, but the difference observed was not significant ( $p=0.406$ ); among HT users, those taking estrogen alone had an increased weight gain compared to women taking estrogen with progestin 1775 vs $24 \mathrm{gl}$, although no statistically significant difference was observed and the same applied when comparing the dose initially prescribed (92 vs $49 \mathrm{~g}$ ). CONCLUSIONS: despite the common belief about weight gain associated with $H T$, the results of the present study seem to contradict this point, with no additional weight gain beyond that normally associated with this period in a woman's life.

\section{Correspondêncie \\ Fernanda Águos \\ Serviço de Ginecologia Obstetríicia Maternidade Bissaya Barreto Rua Augusta, 3000 Coimbra, Portugal \\ Recebido $06 / 06 / 2011$ \\ Aceito com modificacoêes}

Serviço de Ginecologia da Maternidade Bissaya Barreto, Centro Hospitalar de Coimbra, Coimbra, Portugal

1 Interno Complementar de Ginecologia Obstetrícia da Maternidade Bissaya Barreto, Coimbra, Portugal

${ }^{2}$ Assistente graduado de Ginecologia Obstetrícia da Maternidade Bissaya Barreto, Coimbra, Portugal

${ }^{3}$ Chefe de Serviço de Ginecologia Obstetrícia da Maternidade Bissaya Barreto, Coimbra, Portugal 
Introdução

O declínio de estrogênios circulantes na perimenopausa induz assiduamente sintomatologia de difícil tolerabilidade que afeta o bem-estar da mulher. A terapia hormonal (TH) assume-se como tratamento eficaz ${ }^{1}$, com benefícios acrescidos que transcendem o mero alívio de sintomatologia vasomotora.

No entanto, o problema da adesão à referida terapia é reconhecido como relevante. Acredita-se que somente $30 \%$ das mulheres a quem se prescreve terapia hormonal (formulações estrogênicas isoladas ou estroprogestagênicas) mantêm o tratamento por mais de 3 anos, com fatores como a falta de informação, o receio de câncer e crença de ganho ponderal assiduamente referidos como responsáveis por esse fato ${ }^{1,2}$.

Frequentemente, a preocupação feminina em torno do aumento ponderal é puramente cosmética, embora aspectos relacionados ao risco acrescido de doença cardiovascular ${ }^{3} \mathrm{e}$ de câncer da mama ou endométrio ${ }^{4}$ comecem a representar preocupações potenciais da mulher nesta etapa da vida. Mas terão os estrogênios um papel regulador no peso corporal feminino? A evidência indica que, associado ao declínio de estrogênios endógenos, a taxa metabólica basal diminui nos anos perimenopausa, resultando na redução da utilização de calorias com o consecutivo aumento de peso $^{5,6}$. Associadamente, parece haver uma redistribuição da gordura corporal no referido período, passando a mulher da típica forma feminina para uma forma corporal mais andrógena, com adiposidade central, em oposição a uma forma corporal ginoide com adiposidade periférica, típica do período pré-menopáusico.

Estudos em animais de laboratório evidenciaram que os estrogênios desempenham um importante papel na regulação do peso corporal. A ooforectomia foi assídua e consistentemente associada a ganho ponderal, fato atenuado pela reposição estrogênica. Pareceu haver múltiplos mecanismos na base do ganho de peso pela deficiência de estrogênios em animais, incluindo maior aporte alimentar, diminuição espontânea da atividade física e da taxa metabólica. Se os mesmos mecanismos ocorrerem em humanos, isto poderia sugerir a existência de uma unidade biológica na perimenopausa que impulsiona o ganho ponderal ${ }^{7}$. Face a menor taxa metabólica, para se obter o mesmo gasto energético é exigido um maior tempo de atividade física.

A menopausa parece, pois, induzir uma obesidade androide que propicia acrescido risco cardiovascular. Com efeito, embora fatores ambientais como tabagismo, atividade/sedentarismo, stress, resistência à insulina possam interferir no padrão de gordura corporal, as diferenças na redistribuição de gordura associadas ao sexo são largamente atribuídas a hormônios esteroides circulantes ${ }^{8}$.
Acredita-se que o desequilíbrio estrogênico seja a causa mais provável de síndromes metabólicas durante a perimenopausa, contribuindo para o aumento de peso e risco adicional de hipertensão, alterações lipídicas próaterogênicas, aumento da resistência a insulina, diabetes e doença cardiovascular?.

Apesar da divulgação popular comum de que a TH implique aumento ponderal, as evidências correntes e atuais parecem contrariar esta suposta propensão ${ }^{10}$, com a TH parecendo assumir papel preventivo na adiposidade central, atenuando as tendências descritas ${ }^{11-15}$ e transformando a crença popular num possível mito que ganha consistência na medida que, sendo um tema indubitavelmente controverso ${ }^{14}$, os autores não encontraram na literatura estudos que testemunhem ou comprovem essa associação.

Com o estudo efetuado na nossa instituição, pretendeu-se avaliar o impacto da Terapia Hormonal no peso de mulheres na perimenopausa, bem como o efeito de diferentes regimes terapêuticos no referido parâmetro.

\section{Métodos}

Procedeu-se à análise retrospectiva de mulheres, com menopausa recente, referenciadas à consulta de climatério do nosso Departamento no segundo semestre de 2008 para avaliação inicial, acompanhamento e ponderação de terapia hormonal. Os critérios de inclusão basearam-se em mulheres com menopausa há menos de 2 anos, sem terapêutica de reposição hormonal até ao momento da primeira consulta, sem alterações do estilo de vida (dieta ou atividade física) durante o período anual avaliado e sem diabetes associado.

Das mulheres elegíveis ao estudo, foram estabelecidos dois grupos: mulheres a quem se iniciou terapia hormonal (TH) nessa primeira consulta e outro, grupo controle, a quem não foi prescrita farmacoterapia hormonal (por opção da mulher ou por contraindicação à terapêutica). Em cada grupo, foi avaliada a modificação ponderal no intervalo de 1 ano após a primeira consulta. Nas submetidas a TH, avaliou-se esse mesmo parâmetro em função de diferentes regimes terapêuticos preconizados: estrogêneo isolado vs estroprogestativo e dose standard vs baixa dosagem, com dose standard correspondendo a regimes farmacológicos isolados ou combinados integrando $2 \mathrm{mg}$ de valerato de estradiol ou $2 \mathrm{mg}$ de estradiol ( via oral) ou sistemas libertando $50 \mu \mathrm{g}$ estradiol (via transdérmica).

A análise estatística foi realizada pelo programa SPSS versão 17. Para a comparação da distribuição das variáveis idade, idade da menopausa, IMC, peso basal entre grupos foi, utilizado o Teste $t$ de Student, com os resultados expressos em média e desvio padrão. Quanto ao tipo de menopausa, medicação antidepressiva associada a 
cada grupo, utilizou-se o teste de proporção simples com valores expressos em porcentagem. Foi utilizado o teste ANOVA para a comparação das variações de médias ponderais entre os grupos. Foi considerado estatisticamente significante $\mathrm{p}<0,05$.

\section{Resultados}

O estudo incluiu 139 mulheres, 89 das quais alvo de prescrição de terapia hormonal numa primeira consulta de climatério (Gth-grupo com terapêutica hormonal) e 50 a quem não foi facultada terapia hormonal (Gsth - grupo controle sem terapêutica hormonal).

Quando avaliadas características inerentes aos dois grupos não foram verificadas diferenças significativas, tratando-se de grupos similares no que diz respeito a idade, idade média da menopausa, IMC, tipo de menopausa, medicação antidepressiva associada e exercício físico regular (Tabela 1). Comparamos o peso basal de ambos os grupos na primeira consulta e verificamos valores ponderais idênticos.

Quando comparamos o ganho ponderal médio um ano após a primeira consulta, verificamos um ganho médio acrescido no grupo controle face ao grupo sob terapia hormonal (434 g versus $76 \mathrm{~g}$ ), embora diferenças sem valor estatístico ( $\mathrm{p}=0,406$-pns).

Visando avaliar efeitos de diferentes regimes terapêuticos, foram avaliadas as usuárias de terapia hormonal, dividindo aquelas sob estrogênio isolado $(n=11)$ e aquelas sob estroprogestativo $(\mathrm{n}=78)$, com aumento ponderal médio superior no primeiro grupo (775 vs $24 \mathrm{~g}$ ), diferenças não significativas (pns). Relativamente à dosagem hormonal inicialmente prescrita, 48 mulheres foram medicadas com terapia hormonal de dosagem standard e 41 medicadas com baixa dose. No período analisado, houve ganho ponderal médio de $92 \mathrm{~g}$ no primeiro grupo face a $49 \mathrm{~g}$ no segundo, diferenças sem valor estatístico significativo (pns).

\section{Discussão}

Apesar da crença comumente partilhada do aumento ponderal associado à Terapia Hormonal, e que segundo a literatura está associada a cerca de $20 \%$ da falta de adesão a esta, os resultados do nosso estudo parecem contrariar esse aspecto, não havendo um ganho ponderal adicional ao normalmente associado a este período da vida da mulher, caracterizado por uma redução marcada da taxa metabólica. Com efeito, apesar de discretos, os efeitos do seu consumo parecem atenuar o aumento ponderal, com as nossas mulheres-alvo de terapêutica a apresentar menor ganho de peso. A ideia de que farmacoterapia de maior dosagem teria reflexo nesse parâmetro viria a não ser comprovada
Tabela 1. Caracterização da amostra - comparação de características grupais

\begin{tabular}{lcccc}
\hline & \multicolumn{3}{c}{ Grupo Gth } & \multicolumn{2}{c}{ Grupo Gsth } \\
\cline { 2 - 5 } & Média & $\begin{array}{c}\text { Desvio } \\
\text { padrão }\end{array}$ & Média & $\begin{array}{c}\text { Desvio } \\
\text { padrão }\end{array}$ \\
\hline Média de idade (anos) & 53,8 & 4,5 & 55,4 & 6,4 \\
Média de idade da menopausa (anos) & 49,6 & 3,7 & 50,1 & 4,8 \\
IMC (kg/m ${ }^{2}$ ) & 24,5 & 2,8 & 24,8 & 3,0 \\
Peso basal (kg) & 70,9 & 11,4 & 72,3 & 13,2 \\
Menopausa espontânea (\%) & \multicolumn{2}{c}{94,3} & \multicolumn{2}{c}{92,1} \\
Medicação antidepressiva (\%) & \multicolumn{2}{c}{14,0} & \multicolumn{2}{c}{16,0} \\
Exercício físico regular (\%) & \multicolumn{2}{c}{37,9} & \multicolumn{2}{c}{40,1} \\
\hline
\end{tabular}

Gth-grupo com terapêutica hormonal; Gsth - grupo controle sem terapêutica hormonal.

pelo estudo, devendo o tipo de dose prescrita ser baseado noutros fatores e não ponderado com base na possível interferência no peso. O uso do estrogênio isolado também não mostrou ter influência relevante no ganho ponderal face a fármacos com associação estroprogestativa.

A nossa investigação demonstrou sintonia com vários estudos randomizados na literatura ${ }^{16,17}$, que descrevem um ganho ponderal atenuado em mulheres com terapêutica hormonal face a mulheres sem terapia hormonal, conferindo à referida terapia um efeito modesto, mas detectável, na redução do ganho ponderal previsível nos primeiros anos deste importante período da vida feminina. Já quanto ao tipo de formulação ou a dose inicialmente prescrita, em que a nossa análise não demonstrou diferenças significativas no ganho ponderal, encontramos estudos do Women's Health Iniciative (WHI) que revelam aumento ponderal mais marcado e com relevância estatística nas mulheres com terapêutica estrogênica isolada face a sua associação com progestativo ${ }^{14}$.

As alterações metabólicas resultantes da transição menopáusica modificam dramaticamente o ambiente hormonal feminino ${ }^{12}$. A etiologia da obesidade perimenopausa não está perfeitamente estabelecida, permanecendo incerto se o ganho ponderal acrescido e distribuição da gordura corporal associado a este período resultam de alterações climatéricas ou estão simplesmente relacionadas com o envelhecimento individual e/ou alterações do estilo de vida ${ }^{6,18}$.

Com efeito, perda de massa muscular, declínio no metabolismo aeróbio máximo, diminuição de massa magra caracterizam o processo de envelhecimento. A menor taxa metabólica e gastos energéticos decorrentes podem traduzir-se em ganho ponderal, salvo se alterações comportamentais forem adotadas ${ }^{7}$. É consensual que o exercício físico pode prevenir o ganho ponderal nesta faixa etária, mas fatores relacionados com idade e menopausa tornam a manutenção de peso um verdadeiro desafio. Assim, mesmo que o exercício regular não garanta perda ponderal, estudos prospectivos indicam que mulheres climatéricas mais ativas têm menor ganho de peso. Assim, apesar do 
menor impacto do exercício neste período, a mulher deve ser incentivada e não desmotivar, dados os vastos benefícios inerentes que transcendem a mera perda ponderal. Estudos recentes revelaram que terapia hormonal associada a exercício físico regular atenua a acumulação de gordura particularmente na região abdominal ${ }^{7,19}$.

Investigações adicionais atribuem à terapia hormonal papel no aumento dos níveis séricos de leptina, (caracteristicamente diminuídos no período pós menopausa), aspecto que se assume como fator contributivo na manutenção do peso corporal e sobretudo na distribuição da gordura corporal ${ }^{20}$. Com relação à sensibilidade à insulina, esta parece estar diminuída na menopausa, condicionando risco acrescido de diabetes e doença cardiovascular, que podem ser parcialmente explicados por alteração ponderal e do padrão de gordura corporal. $\mathrm{Na}$ literatura, são mencionados estudos que demonstram que a terapêutica hormonal pode melhorar a sensibilidade à insulina sem afetar a composição corporal, beneficiando o metabolismo dos lípideos e carbohidratos ${ }^{21-23}$, havendo estudos demonstrativos da menor prevalência da diabetes nas usuárias de $\mathrm{TH}^{14}$.

Como conclusão, deve ser enfatizado que a prevenção do ganho ponderal deve assumir-se como um dos objetivos fundamentais na abordagem da transição climatérica. $\mathrm{O}$ clínico, projetando essa previsível tendência, deve enfatizar mudanças no estilo de vida da mulher ${ }^{24}$, promovendo medidas higienodietéticas, encorajando a atividade física ${ }^{25}$ e ajustando o aporte calórico às menores necessidades nesta faixa etária. Estratégias terapêuticas combinadas parecem minimizar fatores de risco e melhorar a qualidade de vida ${ }^{11,26}$.

Orientar a mulher quanto aos efeitos da menopausa per si no aspecto ponderal e distribuição de gordura corporal e esclarecê-la dos potenciais efeitos da terapia hormonal poderá resultar num aumento da adesão à terapêutica, permitindo à mulher desfrutar dos potenciais efeitos terapêuticos e, consecutivamente, dos seus benefícios máximos.

\section{Referências}

1. Junge W, El-Samalouti V, Gerlinger C, Schaefers M. Effects of menopausal hormone therapy on hemostatic parameters, blood pressure, and body weight: open-label comparison of randomized treatment with estradiol plus drospirenone versus estradiol plus norethisterone acetate. Eur J Obstet Gynecol Reprod Biol. 2009; 147(2): 195-200

2. Reynolds RF, Obermeyer CM, Walker AM, Guilbert D. The role of treatment intentions and concerns about side effects in women's decision to discontinue postmenopausal hormone therapy. Maturitas. 2002;43(3): 183-94.

3. Donahue RP, Abbott RD, Bloom E, Reed DM, Yano K. Central obesity and coronary heart disease in men. Lancet. 1987;1 (8537):821-4.

4. Magnusson C, Baron J, Persson I, Wolk A, Bergstrom R, Trichopoulos $D$, et al. Body size in different periods of life and breast cancer risk in post-menopausal women. Int J Cancer. 1998;76(1):29-34.

5. Poehlman ET, Goran MI, Gardner AW, Ades PA, Arciero PJ, Katzman-Rooks SM, et al. Determinants of decline in resting metabolic rate in aging females. Am J Physiol. 1993;264(3 Pt 1):E450-5.

6. Augoulea A, Mastorakos G, Lambrinoudaki I, Christodoulakos $G$, Creatsas $G$. Role of postmenopausal hormone replacement therapy on body fat gain and leptin levels. Gynecol Endocrinol. 2005;20(4):227-35.

7. Kohrt WM. Exercise, weight gain, and menopause. Mayfield Heights: The North American Menopause Society; 2009.

8. Sumino H, Ichikawa S, Yoshida A, Murakami M, Kanda T, Mizunuma $\mathrm{H}$, et al. Effects of hormone replacement therapy on weight, abdominal fat distribution, and lipid levels in Japanese postmenopausal women. Int J Obes Relat Metab Disord. 2003;27(9):1044-51.

9. Polotsky HN, Polotsky AJ. Metabolic implications of menopause. Semin Reprod Med. 2010;28(5):426-34.

10. Van den Brûle F, Gaspard U. Body mass changes at menopause: impact of therapeutic strategies. Rev Med Liege. 2003;58(12):734-40.
11. Poehlman ET. Menopause, energy expenditure, and body composition. Acta Obstet Gynecol Scand. 2002;81 (7):603-1 1.

12. Tchernof A, Poehlman ET, Després JP. Body fat distribution, the menopause transition, and hormone replacement therapy. Diabetes Metab. 2000;26(1):12-20.

13. Genazzani AR, Gambacciani M. Effect of climacteric transition and hormone replacement therapy on body weight and body fat distribution. Gynecol Endocrinol. 2006;22(3):145-50.

14. Ravnikar VA. Does hormone therapy keep you lean or fat? Menopause. $2011 ; 18(1): 13-4$.

15. Bea JW, Zhao Q, Cauley JA, LaCroix AZ, Bassford T, Lewis CE, et al. Effect of hormone therapy on lean body mass, falls and fractures: six-year results from the Women's Health Initiative Hormone Trials. Menopause. $2011 ; 18(1): 44-52$.

16. Utian WH, Gass ML, Pickar JH. Body mass index does not influence response to treatment, nor does body weight change with lower doses of conjugated estrogens and medroxyprogesterone acetate in early postmenopausal women. Menopause. 2004; 11 (3):30614.

17. Blümel JE, Castelo-Branco C, Rocangliolo ME, Bifa L, Tacla X, Mamani L. Changes in body mass index around menopause: a population study of Chilean woman. Menopause. 2001;8(4):23944.

18. Milewicz A, Tworowska U, Demissie M. Menopausal obesity: myth or fact? Climacteric. 2001;4(4):273-83.

19. Kohrt WM, Ehsani AA, Birge SJ Jr. HRT preserves increases in bone mineral density and reductions in body fat after a supervised exercise program. J Appl Physiol. 1998;84(5):1506-12.

20. Dedeoglu EN, Erenu M, Yoruk P. Effects of hormone therapy and tibolone on body composition and serum leptin levels in postmenopausal women. Fertil Steril. 2009;91 (2):425-31. 
21. Sumino $H$, Ichikawa $S$, Itoh $H$, Utsugi $T$, Ohyama $Y$, Umeda $M$, et al. Hormone replacement therapy decreases insulin resistance and lipid metabolism in Japanese postmenopausal women with impaired and normal glucose tolerance. Horm Res. 2003;60(3): 134-42.

22. Sites CK, L'Hommedieu GD, Toth M, Brochu M, Cooper BC, Fairhurst PA. The effect of hormone replacement therapy on body composition, body fat distribution, and insulin sensitivity in menopausal women: a randomized, double-blind, placebocontrolled trial. J Clin Endocrinol Metab. 2005;90(5):2701-7.
23. Lobo RA. Metabolic syndrome after menopause and the role of hormones. Maturitas. 2008;60(1):10-8.

24. Lovejoy JC. The menopause and obesity. Prim Care. 2003;30(2):317-25.

25. Sénéchal M, Arguin H, Bouchard D, Carpentier AC, Ardilouze JL, Dionne IJ, et al. Weight gain since menopause and its associations with weight loss maintenance in obese postmenopausal women. Clin Interv Aging. $2011 ;(6): 221-5$.

26. Simkin-Silverman $L R$, Wing RR. Weight gain during menopause. Is it inevitable or can it be prevented? Postgrad Med. 2000;108(3):47-50. 